\title{
THE PROPAGATION PHENOMENON OF WEIGHTED SHIFTS
}

\author{
An-Hyun Kim And Eun-Young Kwon
}

\begin{abstract}
This paper concerns the propagation phenomenon of weighted shifts. We here establish the existence of positive real numbers $b$ and $c(1<b<c)$ such that the recursive weighted shift $W_{1,(1, \sqrt{b}, \sqrt{c})^{\wedge}}$ is quadratically but not cubically hyponormal.
\end{abstract}

\section{Introduction}

Let $\mathcal{H}$ and $\mathcal{K}$ be infinite dimensional complex Hilbert spaces, let $\mathcal{B}(\mathcal{H}, \mathcal{K})$ be the set of bounded linear operators from $\mathcal{H}$ to $\mathcal{K}$ and write $\mathcal{B}(\mathcal{H}):=\mathcal{B}(\mathcal{H}, \mathcal{H})$. An operator $T \in \mathcal{B}(\mathcal{H})$ is said to be normal if $T^{*} T=T T^{*}$, hyponormal if $T^{*} T \geq T T^{*}$, and subnormal if $T=\left.N\right|_{\mathcal{H}}$, where $N$ is normal on some Hilbert space $\mathcal{K} \supseteq \mathcal{H}$. Recall that given a bounded sequence of positive numbers $\alpha: \alpha_{0}, \alpha_{1}, \ldots$ (called weights), the (unilateral) weighted shift $W_{\alpha}$ associated with $\alpha$ is the operator on $\ell^{2}\left(\mathbb{Z}_{+}\right)$defined by $W_{\alpha} e_{n}:=\alpha_{n} e_{n+1}$ for all $n \geq 0$, where $\left\{e_{n}\right\}_{n=0}^{\infty}$ is the canonical orthonormal basis for $\ell^{2}$. It is straightforward to check that $W_{\alpha}$ is hyponormal if and only if $\alpha_{n} \leq \alpha_{n+1}$ for all $n \geq 0$. The Bram-Halmos criterion for subnormality states that an operator $T$ is subnormal if and only if (cf. [4, II.1.9])

$$
\left(\begin{array}{cccc}
I & T^{*} & \ldots & T^{* k} \\
T & T^{*} T & \ldots & T^{* k} T \\
\vdots & \vdots & \ddots & \vdots \\
T^{k} & T^{*} T^{k} & \ldots & T^{* k} T^{k}
\end{array}\right) \geq 0 \quad(\text { all } k \geq 1)
$$

Let $[A, B]:=A B-B A$ denote the commutator of two operators $A$ and $B$, and define $T$ to be $k$-hyponormal whenever the $k \times k$ operator matrix

$$
M_{k}(T):=\left(\left[T^{* j}, T^{i}\right]\right)_{i, j=1}^{k}
$$

Received January 2, 2009.

2000 Mathematics Subject Classification. Primary 47B20, 47B37.

Key words and phrases. weighted shifts, subnormal, $k$-hyponormal, quadratically hyponormal, cubically hyponormal.

This research was financially supported by Changwon National University in 2008. 
is positive. An application of the Choleski algorithm for operator matrices shows that the positivity of $(0.2)$ is equivalent to the positivity of the $k+$ $1) \times(k+1)$ operator matrix in $(0.1)$; the Bram-Halmos criterion can be then rephrased as saying that $T$ is subnormal if and only if $T$ is $k$-hyponormal for every $k \geq 1([11])$.

Recall ([1], [5], [11]) that $T \in \mathcal{B}(\mathcal{H})$ is said to be weakly k-hyponormal if $\alpha_{1} T+\alpha_{2} T^{2}+\cdots+\alpha_{k} T^{k}$ is hyponormal for each $\left(\alpha_{1}, \ldots, \alpha_{k}\right) \in \mathbb{C}^{k}$, or equivalently, $M_{k}(T)$ is weakly positive, i.e., ([11])

$$
\left\langle M_{k}(T)\left(\begin{array}{c}
\lambda_{1} x \\
\vdots \\
\lambda_{k} x
\end{array}\right),\left(\begin{array}{c}
\lambda_{1} x \\
\vdots \\
\lambda_{k} x
\end{array}\right)\right\rangle \geq 0 \quad \text { for } x \in \mathcal{H} \text { and } \lambda_{1}, \ldots, \lambda_{k} \in \mathbb{C} .
$$

If $k=2$, then $T$ is said to be quadratically hyponormal, and if $k=3$, then $T$ is said to be cubically hyponormal. Similarly, $T \in \mathcal{B}(\mathcal{H})$ is said to be polynomially hyponormal if $p(T)$ is hyponormal for every polynomial $p \in \mathbb{C}[z]$. It is known that $k$-hyponormal $\Rightarrow$ weakly $k$-hyponormal, but the converse is not true in general.

In this paper we consider a propagation phenomenon of the cubic hyponormality. Although examples abound of nontrivial quadratically hyponormal recursive shifts with two equal weights (cf. [9]), we conjecture that the same is not true for cubic hyponormality. To this end, we show in Theorem 3 the existence of positive numbers $b$ and $c$ such that the recursive shift $W_{1,(1, \sqrt{b}, \sqrt{c})^{\wedge}}$ is quadratically hyponormal but not cubically hyponormal. This gives an explicit description of the gap between quadratic hyponormality and cubic hyponormality for recursive shifts.

\section{The main result}

J. Stampfli [15] showed that for subnormal weighted shifts $W_{\alpha}$, a propagation phenomenon occurs which forces the flatness of $W_{\alpha}$ whenever two equal weights are present. Later, A. Joshi proved in [13] that the shift with weights $\alpha_{0}=\alpha_{1}=a, \alpha_{2}=\alpha_{3}=\cdots=b, 0<a<b$, is not quadratically hyponormal, and P. Fan [12] established that for $a=1, b=2$, and $0<s<\sqrt{5} / 5$, $W_{\alpha}+s W_{\alpha}^{2}$ is not hyponormal. On the other hand, it was shown in $[6$, Theorem 2] that a hyponormal weighted shift with three equal weights cannot be quadratically hyponormal without being flat: If $W_{\alpha}$ is quadratically hyponormal and $\alpha_{n}=\alpha_{n+1}=\alpha_{n+2}$ for some $n \geq 0$, then $\alpha_{1}=\alpha_{2}=\alpha_{3}=\cdots$, i.e., $W_{\alpha}$ is subnormal. Furthermore, in [6, Proposition 11] it was shown that, in the presence of quadratic hyponormality, two consecutive pairs of equal weights again force flatness, thereby subnormality.

Theorem 1 (Propagation). Let $W_{\alpha}$ be a weighted shift with weight sequence $\left\{\alpha_{n}\right\}_{n=0}^{\infty}$. 
(i) ([15, Theorem 6]) Let $W_{\alpha}$ be subnormal. If $\alpha_{n}=\alpha_{n+1}$ for some $n \geq 0$, then $\alpha$ is flat, i.e., $\alpha_{1}=\alpha_{2}=\alpha_{3}=\cdots$.

(ii) ([6, Corollary 6]) Let $W_{\alpha}$ be 2-hyponormal. If $\alpha_{n}=\alpha_{n+1}$ for some $n \geq 0$, then $\alpha$ is flat.

(iii) ([2, Theorem 1]) Let $W_{\alpha}$ be quadratically hyponormal. If $\alpha_{n}=\alpha_{n+1}$ for some $n \geq 1$, then $\alpha$ is flat.

Before we proceed, we consider the selfcommutator $\left[\left(W_{\alpha}+s W_{\alpha}^{2}\right)^{*}, W_{\alpha}+\right.$ $\left.s W_{\alpha}^{2}\right]$. Let $W_{\alpha}$ be a hyponormal weighted shift. For $s \in \mathbb{C}$, we write

$$
D(s):=\left[\left(W_{\alpha}+s W_{\alpha}^{2}\right)^{*}, W_{\alpha}+s W_{\alpha}^{2}\right]
$$

and we let

$$
D_{n}(s):=P_{n}\left[\left(W_{\alpha}+s W_{\alpha}^{2}\right)^{*}, W_{\alpha}+s W_{\alpha}^{2}\right] P_{n}=\left(\begin{array}{ccccccc}
q_{0} & \bar{r}_{0} & 0 & \ldots & 0 & 0 \\
r_{0} & q_{1} & \bar{r}_{1} & \ldots & 0 & 0 \\
0 & r_{1} & q_{2} & \ldots & 0 & 0 \\
\vdots & \vdots & \vdots & \ddots & \vdots & \vdots \\
0 & 0 & 0 & \ldots & q_{n-1} & \bar{r}_{n-1} \\
0 & 0 & 0 & \ldots & r_{n-1} & q_{n}
\end{array}\right),
$$

where $P_{n}$ is the orthogonal projection onto the subspace generated by $\left\{e_{0}, \ldots\right.$, $\left.e_{n}\right\}$

$$
\left\{\begin{array}{l}
q_{n}:=u_{n}+|s|^{2} v_{n} \\
r_{n}:=s \sqrt{w_{n}} \\
u_{n}:=\alpha_{n}^{2}-\alpha_{n-1}^{2} \\
v_{n}:=\alpha_{n}^{2} \alpha_{n+1}^{2}-\alpha_{n-1}^{2} \alpha_{n-2}^{2} \\
w_{n}:=\alpha_{n}^{2}\left(\alpha_{n+1}^{2}-\alpha_{n-1}^{2}\right)^{2}
\end{array}\right.
$$

and, for notational convenience, $\alpha_{-2}=\alpha_{-1}=0$. Clearly, $W_{\alpha}$ is quadratically hyponormal if and only if $D_{n}(s) \geq 0$ for all $s \in \mathbb{C}$ and all $n \geq 0$. Let $d_{n}(\cdot):=$ $\operatorname{det}\left(D_{n}(\cdot)\right)$. Then $d_{n}$ satisfies the following 2 -step recursive formula:

$$
d_{0}=q_{0}, \quad d_{1}=q_{0} q_{1}-\left|r_{0}\right|^{2}, \quad d_{n+2}=q_{n+2} d_{n+1}-\left|r_{n+1}\right|^{2} d_{n} .
$$

If we let $t:=|s|^{2}$, we observe that $d_{n}$ is a polynomial in $t$ of degree $n+1$, and if we write $d_{n} \equiv \sum_{i=0}^{n+1} c(n, i) t^{i}$, then the coefficients $c(n, i)$ satisfy a doubleindexed recursive formula, namely

(1.4)

$$
\begin{aligned}
c(n+2, i) & =u_{n+2} c(n+1, i)+v_{n+2} c(n+1, i-1)-w_{n+1} c(n, i-1), \\
c(n, 0) & =u_{0} \cdots u_{n}, \quad c(n, n+1)=v_{0} \cdots v_{n}, \quad c(1,1)=u_{1} v_{0}+v_{1} u_{0}-w_{0}
\end{aligned}
$$

$(n \geq 0, i \geq 1)$. We say that $W_{\alpha}$ is positively quadratically hyponormal if $c(n, i) \geq 0$ for every $n \geq 0,0 \leq i \leq n+1$ (cf. [7]). Evidently, positively quadratically hyponormal $\Rightarrow$ quadratically hyponormal. The converse, however, is not true in general (cf. [3]). 
The idea of the proof of Theorem 1 (iii) is based on the following observation: if $W_{\alpha}$ is quadratically hyponormal with $\alpha_{1}=\alpha_{2}=1$, then a straightforward calculation shows that

$$
d_{4}(t)=\alpha_{0}^{2} \alpha_{4}^{2}\left(\alpha_{0}^{2}-1\right)\left(\alpha_{3}^{2}-1\right)^{3} t^{2}+c(4,3) t^{3}+c(4,4) t^{4}+c(4,5) t^{5},
$$

so

$$
\lim _{t \rightarrow 0+} \frac{d_{4}(t)}{t^{2}}=\alpha_{0}^{2} \alpha_{4}^{2}\left(\alpha_{0}^{2}-1\right)\left(\alpha_{3}^{2}-1\right)^{3} \geq 0
$$

which forces $\alpha_{0}=1$ or $\alpha_{3}=1$, so that three equal weights are present and hence by [6, Theorem 2], flatness occurs.

Note that in Theorem 1 (iii) the condition " $n \geq 1$ " cannot be relaxed to " $n \geq 0$ ". For example, if (cf. [6, Proposition 7])

$$
\alpha_{0}=\alpha_{1}=\sqrt{\frac{2}{3}}, \quad \alpha_{n}=\sqrt{\frac{n+1}{n+2}}(n \geq 2),
$$

then $W_{\alpha}$ is quadratically hyponormal but not cubically hyponormal (and hence not subnormal); indeed if we let

$$
C_{5}(t):=\operatorname{det}\left(P_{5}\left[\left(W_{\alpha}+t W_{\alpha}^{2}+t^{2} W_{\alpha}^{3}\right)^{*},\left(W_{\alpha}+t W_{\alpha}^{2}+t^{2} W_{\alpha}^{3}\right)\right] P_{5}\right)
$$

then

$$
\lim _{t \rightarrow 0+} \frac{C_{5}(t)}{t^{8}}=-\frac{1}{2041200}<0
$$

We briefly pause to recall that when $\alpha_{0}=\alpha_{1}=1$, quadratic hyponormality implies

$$
\alpha_{2}<\sqrt{2} \text { and } \quad \alpha_{3} \geq\left(2-\alpha_{2}^{2}\right)^{-2}
$$

(cf. [7, p. 78]).

At this point one might guess that every cubically hyponormal weighted shift with two equal weights is subnormal. To affirm this, it would suffice to show, in view of Theorem 1 (iii), that if $W_{\alpha}$ is cubically hyponormal and if $\alpha_{0}=\alpha_{1}$, then $W_{\alpha}$ is flat.

In the sequel, given $\alpha_{0}<\alpha_{1}<\alpha_{2}$ we denote by $W_{\left(\alpha_{0}, \alpha_{1}, \alpha_{2}\right)^{\wedge}}$ the recursive weighted shift whose weights are calculated according to the recursive relation

$$
\alpha_{n+1}^{2}=\varphi_{1}+\varphi_{0} \frac{1}{\alpha_{n}^{2}}
$$

where

$$
\varphi_{0}=-\frac{\alpha_{0}^{2} \alpha_{1}^{2}\left(\alpha_{2}^{2}-\alpha_{1}^{2}\right)}{\alpha_{1}^{2}-\alpha_{0}^{2}} \quad \text { and } \quad \varphi_{1}=\frac{\alpha_{1}^{2}\left(\alpha_{2}^{2}-\alpha_{0}^{2}\right)}{\alpha_{1}^{2}-\alpha_{0}^{2}} .
$$

It is well-known that $W_{\left(\alpha_{0}, \alpha_{1}, \alpha_{2}\right)}$ is subnormal with 2-atomic Berger measure. Let $W_{x}\left(\alpha_{0}, \alpha_{1}, \alpha_{2}\right)^{\wedge}$ denote the weighted shift whose weight sequence consists of the initial weight $x$ followed by the weight sequence of $W_{\left(\alpha_{0}, \alpha_{1}, \alpha_{2}\right)^{\wedge}}$. In [8], it was shown that there exists $1<b<c$ such that $W_{1,(1, \sqrt{b}, \sqrt{c})^{\wedge}}$ is quadratically hyponormal. 
In this stage we would pose:

Question 2. Does there exist $1<b<c$ such that $W_{1,(1, \sqrt{b}, \sqrt{c})^{\wedge}}$ is cubically hyponormal?

In [10, Example 3.1] it was shown that $W_{\sqrt{x},(\sqrt{a}, \sqrt{b}, \sqrt{c})^{\wedge}}$ is 2-hyponormal if and only if it is subnormal. Thus one might guess that $W_{1,(1, \sqrt{b}, \sqrt{c})^{\wedge}}$ is quadratically hyponormal if and only if it is polynomially hyponormal. However, the 2-hyponormality of $W_{\sqrt{x},(\sqrt{a}, \sqrt{b}, \sqrt{c})^{\wedge}}$ never admits $a$ as a value for $x$. By contrast, quadratic hyponormality does admit the value $a$. Thus, the situation for weak $k$-hyponormality becomes more delicate. In view of the preceding considerations, we conjecture that the answer to Question 2 is negative; the following theorem provides a strong evidence.

Theorem 3. There exists $1<b<c$ such that $W_{1,(1, \sqrt{b}, \sqrt{c})^{\wedge}}$ is quadratically hyponormal but not cubically hyponormal.

Proof. Let $W_{\alpha}$ be a hyponormal weighted shift with weight sequence $\left\{\alpha_{n}\right\}_{n=0}^{\infty}$. For $s, t \in \mathbb{C}$ we let

$$
C_{n}(s, t):=P_{n}\left[\left(W_{\alpha}+s W_{\alpha}^{2}+t W_{\alpha}^{3}\right)^{*}, W_{\alpha}+s W_{\alpha}^{2}+t W_{\alpha}^{3}\right] P_{n}
$$

$C_{n}(s, t)$ is a pentadiagonal matrix:

$$
C_{n}(s, t)=\left(\begin{array}{ccccccccc}
q_{0} & r_{0} & u_{0} & 0 & & & & & \\
\bar{r}_{0} & q_{1} & r_{1} & u_{1} & 0 & & & & \\
\bar{u}_{0} & \bar{r}_{1} & q_{2} & r_{2} & u_{2} & 0 & & & \\
0 & \bar{u}_{1} & \bar{r}_{2} & q_{3} & r_{3} & u_{3} & \ddots & & \\
& \ddots & \ddots & \ddots & \ddots & \ddots & \ddots & \ddots & \\
& & \ddots & \ddots & \ddots & \ddots & \ddots & \ddots & 0 \\
& & & \ddots & \ddots & \ddots & \ddots & \ddots & u_{n-2} \\
& & & & \ddots & \ddots & \ddots & \ddots & r_{n-1} \\
& & & & & 0 & \bar{u}_{n-2} & \bar{r}_{n-1} & q_{n}
\end{array}\right),
$$

where

$$
\begin{aligned}
q_{n}:= & \left(\alpha_{n}^{2}-\alpha_{n-1}^{2}\right)+\left(\alpha_{n}^{2} \alpha_{n+1}^{2}-\alpha_{n-2}^{2} \alpha_{n-1}^{2}\right)|s|^{2} \\
& \quad+\left(\alpha_{n}^{2} \alpha_{n+1}^{2} \alpha_{n+2}^{2}-\alpha_{n-3}^{2} \alpha_{n-2}^{2} \alpha_{n-1}^{2}\right)|t|^{2}, \\
r_{n}:= & \alpha_{n}\left(\alpha_{n+1}^{2}-\alpha_{n-1}^{2}\right) \bar{s}+\alpha_{n}\left(\alpha_{n+1}^{2} \alpha_{n+2}^{2}-\alpha_{n-1}^{2} \alpha_{n-2}^{2}\right) s \bar{t}, \\
u_{n}:= & \alpha_{n} \alpha_{n+1}\left(\alpha_{n+2}^{2}-\alpha_{n-1}^{2}\right) \bar{t},
\end{aligned}
$$

and, for notational convenience, $\alpha_{-3}=\alpha_{-2}=\alpha_{-1}=0$. Then $W_{\alpha}$ is cubically hyponormal if and only if $\operatorname{det} C_{n}(s, t) \geq 0$ for every $s, t \in \mathbb{C}$ and every $n \geq 0$. Put $\alpha_{0}=\alpha_{1}=1$. Then a straightforward calculation shows that

$$
\operatorname{det} C_{4}(s, 0)=\alpha_{2}^{2}\left(\alpha_{2}^{2}-1\right)\left(\alpha_{4}^{2}-\alpha_{3}^{2}\right)\left(\alpha_{3}^{2}\left(2-\alpha_{2}^{2}\right)-1\right) s^{4}\left(1+p_{1}(s)\right)
$$


and

$$
\operatorname{det} C_{4}\left(s, s^{2}\right)=\alpha_{2}^{2} \alpha_{3}^{2}\left(\alpha_{2}^{2}-1\right)\left(\alpha_{3}^{2}-\alpha_{2}^{2}\right)\left(\alpha_{4}^{2}\left(2-\alpha_{3}^{2}\right)-1\right) s^{6}\left(1+p_{2}(s)\right),
$$

where $p_{i}(s)$ is a polynomial in $s$ with $p_{i}(0)=0$ for each $i=1,2$. Therefore, if $W_{\alpha}$ is cubically hyponormal, then

$$
\alpha_{2}^{2}\left(\alpha_{2}^{2}-1\right)\left(\alpha_{4}^{2}-\alpha_{3}^{2}\right)\left(\alpha_{3}^{2}\left(2-\alpha_{2}^{2}\right)-1\right) \geq 0
$$

and

$$
\alpha_{2}^{2} \alpha_{3}^{2}\left(\alpha_{2}^{2}-1\right)\left(\alpha_{3}^{2}-\alpha_{2}^{2}\right)\left(\alpha_{4}^{2}\left(2-\alpha_{3}^{2}\right)-1\right) \geq 0
$$

Thus, if $\left\{\alpha_{n}\right\}_{n=1}^{\infty}$ is strictly increasing, then

$$
\alpha_{k+1}^{2} \geq \frac{1}{2-\alpha_{k}^{2}} \quad \text { and } \quad 1<\alpha_{k}^{2}<2 \quad \text { for } k=2,3 .
$$

Write $\left\{\alpha_{n}\right\}_{n=0}^{\infty}: 1,(1, \sqrt{b}, \sqrt{c})^{\wedge}(1<b<c)$. In view of Theorem 1 (iii), $\left\{\alpha_{n}\right\}_{n=1}^{\infty}$ is strictly increasing. Put

$$
\varphi_{1}:=\frac{b(c-1)}{b-1} \text { and } \varphi_{0}:=-\frac{b(c-b)}{b-1} .
$$

Then $\alpha_{4}^{2}=\varphi_{1}+\frac{\varphi_{0}}{c}=\frac{b\left(c^{2}-2 c+b\right)}{c(b-1)}$. Thus by (3.2), we have that $c \geq \frac{1}{2-b}$ and $\alpha_{4}^{2} \geq \frac{1}{2-c}$, i.e.,

$$
\frac{b\left(c^{2}-2 c+b\right)}{c(b-1)} \geq \frac{1}{2-c} .
$$

Therefore, if $W_{1,(1, \sqrt{b}, \sqrt{c})^{\wedge}}$ is cubically hyponormal, then

$$
g(b, c):=c^{3}-4 c^{2}+\left(b+5-\frac{1}{b}\right) c-2 b \leq 0 .
$$

It is known ([8, Proposition 4.6]) that if $b=\frac{11}{10}$, then there exists a value of $c$ between $\frac{1142}{1000}$ and $\frac{1143}{1000}$ for which $W_{1,(1, \sqrt{b}, \sqrt{c})^{\wedge}}$ is quadratically hyponormal. But a direct calculation shows that $g\left(\frac{11}{10}, c\right)>0$ for $\frac{1142}{1000}<c<\frac{1143}{1000}$, so that the corresponding shift is not cubically hyponormal.

As a strategy to answer Question 2, recall an argument in [8, Theorem 4.3]. Let $0<a<b<c$, let $\alpha: \sqrt{x},(\sqrt{a}, \sqrt{b}, \sqrt{c})^{\wedge}$ and let

$$
h_{2}^{+}:=\left(\sup \left\{x: W_{\alpha} \text { is positively quadratically hyponormal }\right\}\right)^{\frac{1}{2}} \text {. }
$$

As before, write $\varphi_{1}:=\frac{b(c-a)}{b-a}$ and $\varphi_{0}:=-\frac{a b(c-b)}{b-a}$. Put

$$
L^{2}:=\frac{\varphi_{1}+\sqrt{\varphi_{1}^{2}+4 \varphi_{0}}}{2} \text { and } K:=-\frac{\varphi_{1}^{2} L^{2}}{\varphi_{0}} .
$$


In [8], it was shown that

$$
h_{2}^{+}=\min \{\sqrt{a}, \quad f(a, b, c)\},
$$

where

$$
f(a, b, c):=\left(\frac{a^{2} b^{2} c+a b^{2}(c-a) K+a b(c-b) K^{2}}{a^{3} b+a b(c-a) K+\left(a^{2}+b c-2 a b\right) K^{2}}\right)^{\frac{1}{2}} .
$$

Note that the second term under min in (3.5) may be greater than the first: for example if $a=\frac{17}{5}, b=\frac{58}{17}$ and $c=\frac{99}{29}$, then

$$
f(a, b, c) \approx \sqrt{3.4218}>\sqrt{\frac{17}{5}}=\sqrt{a} .
$$

Thus, in this case, $\alpha: \sqrt{\frac{17}{5}},\left(\sqrt{\frac{17}{5}}, \sqrt{\frac{58}{17}}, \sqrt{\frac{99}{29}}\right)^{\wedge}$ induces a positively quadratically hyponormal shift. For $\alpha: \sqrt{x},(\sqrt{a}, \sqrt{b}, \sqrt{c})^{\wedge}$ write

$$
h_{2}:=\left(\sup \left\{x: W_{\alpha} \text { is quadratically hyponormal }\right\}\right)^{\frac{1}{2}} .
$$

Clearly, $h_{2}^{+} \leq h_{2}$. In [14, Theorem 4.6], it was shown that $h_{2} \leq f(a, b, c)$; consequently, we have

$$
h_{2}=\min \{\sqrt{a}, \quad f(a, b, c)\} .
$$

If $1<b<c$ write

$$
\begin{aligned}
& \mathfrak{H}_{2}:=\left\{(b, c): W_{1,(1, \sqrt{b}, \sqrt{c})^{\wedge}} \text { is quadratically hyponormal }\right\} ; \\
& \mathfrak{H}_{3}:=\left\{(b, c): W_{1,(1, \sqrt{b}, \sqrt{c})^{\wedge}} \text { is cubically hyponormal }\right\} .
\end{aligned}
$$

Corollary 4. If $1<b<c$ we let

$$
\begin{aligned}
& f(b, c):=b(b c-1)+b(b-1)(c-1) K-(b-1)^{2} K^{2} \\
& g(b, c):=c^{3}-4 c^{2}+\left(b+5-\frac{1}{b}\right) c-2 b,
\end{aligned}
$$

where $K$ is given by (3.4) with $a=1$. Then we have:

(i) $\mathfrak{H}_{2}=\{(b, c): f(b, c) \geq 0\}$;

(ii) $\mathfrak{H}_{3} \subseteq\{(b, c): f(b, c) \geq 0$ and $g(b, c) \leq 0\}$.

Proof. By (3.6), $W_{1,(1, \sqrt{b}, \sqrt{c})^{\wedge}}$ is quadratically hyponormal if and only if $1 \leq$ $f(a, b, c)$, or equivalently,

$$
b(b c-1)+b(b-1)(c-1) K-(b-1)^{2} K^{2} \geq 0,
$$

where

$$
K=\frac{b(c-1)^{2}\left(b(c-1)+\sqrt{b^{2}(c-1)^{2}-4 b(b-1)(c-b)}\right)}{2(b-1)^{2}(c-b)} .
$$

This proves assertion (i). Assertion (ii) follows from assertion (i) and (3.3). 
Acknowledgements. The authors are thankful to Prof. Woo Young Lee at Seoul National University for helpful suggestions for this topic.

\section{References}

[1] A. Athavale, On joint hyponormality of operators, Proc. Amer. Math. Soc. 103 (1988), no. 2, 417-423.

[2] Y. B. Choi, A propagation of quadratically hyponormal weighted shifts, Bull. Korean Math. Soc. 37 (2000), no. 2, 347-352.

[3] Y. B. Choi, J. K. Han, and W. Y. Lee, One-step extension of the Bergman shift, Proc. Amer. Math. Soc. 128 (2000), no. 12, 3639-3646.

[4] J. B. Conway, The Theory of Subnormal Operators, Math. Surveys and Monographs, vol. 36, Amer. Math. Soc., Providence, 1991.

[5] J. B. Conway and W. Szymanski, Linear combinations of hyponormal operators, Rocky Mountain J. Math. 18 (1988), no. 3, 695-705.

[6] R. E. Curto, Quadratically hyponormal weighted shifts, Integral Equations Operator Theory 13 (1990), no. 1, 49-66.

[7] _ An operator-theoretic approach to truncated moment problems, Linear operators (Warsaw, 1994), 75-104, Banach Center Publ., 38, Polish Acad. Sci., Warsaw, 1997.

[8] R. E. Curto and L. A. Fialkow, Recursively generated weighted shifts and the subnormal completion problem. II, Integral Equations Operator Theory 18 (1994), no. 4, 369-426.

[9] R. E. Curto and I. B. Jung, Quadratically hyponormal weighted shifts with two equal weights, Integral Equations Operator Theory 37 (2000), no. 2, 208-231.

[10] R. E. Curto and W. Y. Lee, Joint hyponormality of Toeplitz pairs, Mem. Amer. Math. Soc. 150 (2001), no. 712, x+65 pp.

[11] R. E. Curto, P. S. Muhly, and J. Xia, Hyponormal pairs of commuting operators, Contributions to operator theory and its applications (Mesa, AZ, 1987), 1-22, Oper. Theory Adv. Appl., 35, Birkhauser, Basel, 1988.

[12] P. Fan, A note on hyponormal weighted shifts, Proc. Amer. Math. Soc. 92 (1984), no. $2,271-272$.

[13] A. Joshi, Hyponormal polynomials of monotone shifts, Indian J. Pure Appl. Math. 6 (1975), no. 6, 681-686.

[14] I. B. Jung and S. S. Park, Quadratically hyponormal weighted shifts and their examples, Integral Equations Operator Theory 36 (2000), no. 4, 480-498.

[15] J. Stampfli, Which weighted shifts are subnormal?, Pacific J. Math. 17 (1966), 367-379.

AN-HYun KIM

Department of Mathematics

Changwon National University

Changwon 641-773, Korea

E-mail address: ahkim@changwon.ac.kr

EUn-YOUnG KWON

EDUCATIONAL INSTITUTE OF ENGINEERING

Changwon National University

Changwon 641-773, Korea

E-mail address: key3506@changwon.ac.kr 